\title{
InGaP/GaAs Inverted Dual Junction Solar Cells For CPV Applications Using Metal-Backed Epitaxial Lift-Off
}

\author{
Gerard J. Bauhuis ${ }^{1}$, Peter Mulder ${ }^{1}$, Erik J. Haverkamp ${ }^{1}$, John J. Schermer ${ }^{1}$, Lee \\ J. Nash ${ }^{2}$, Dominic J.F. Fulgoni ${ }^{2}$, Ian M. Ballard ${ }^{2}$ and Geoffrey Duggan ${ }^{2, i}$. \\ 1. Applied Materials Science, IMM, Radboud University Nijmegen, Toernooiveld 1, 625 ED Nijmegen, The \\ Netherlands \\ 2. Circadian Solar Ltd, One Sovereign Court, Sir William Lyons Road, Coventry, CV4 7EZ, U.K.
}

\begin{abstract}
The epitaxial lift-off (ELO) technique has been combined with inverted III-V PV cell epitaxial growth with the aim of employing thin film PV cells in HCPV systems. In a stepwise approach to the realization of an inverted triple junction on a MELO platform we have first grown a GaAs single junction PV cell to establish the basic layer release process and cell processing steps followed by the growth, fabrication and test of an inverted InGaP/GaAs dual junction structure
\end{abstract}

Keywords: Semiconducting III-V materials, Solar Cells, Etching, Doping.

PACS: 71.55.Eq; 73.61.-r; 81.15.Gh; 82.45.Mp; 84.60.Jd

\section{INTRODUCTION}

If the generation of electricity from CPV systems is to be widely adopted then the cost of generating that electricity has to be reduced from today's levels until it becomes competitive with power generation from fossil fuel sources. To achieve this aim it will be necessary to both increase the efficiency of power generation of the CPV system whilst simultaneously reducing the costs of system production.

The biggest factor in increasing the system efficiency is the performance of the multijunction (MJ) solar cell. The efficiency of MJ solar cells has seen a steady progression of approximately $1-2 \%$ per annum over the last ten years culminating in the recent world record of an efficiency of $41.6 \%$ for a triple junction lattice matched cell grown on a Ge substrate ${ }^{1}$. Reducing the cost of the overall system will be achieved as CPV becomes accepted as a bankable technology and manufacturers benefit from the economies of scale brought about by mass production. As well as playing the most significant part in increasing system efficiency, the III-V MJ cell is also one of the most significant cost contributors to the overall system. The cost of these cells could potentially be significantly reduced through the realization of an inorganic, thin film MJ cell by removal and re-use of the expensive substrate whilst simultaneously maintaining the high conversion efficiency, especially under concentrated sunlight.

Epitaxial Lift Off (ELO) is a technique which allows an epitaxially deposited film to be separated from the substrate on which it was deposited ${ }^{2}$ and the ELO technique has been shown to produce world record single junction, one sun efficiencies ${ }^{3}$. In addition the ELO process is naturally compatible with the growth and fabrication of an inverted metamorphic multijunction (IMM) cells which has also shown itself capable of producing world leading efficiencies under concentration $^{4}$. In Ref. 4 the GaAs substrate was not reused but removed using selective chemical etching. Tatavarti et $\mathrm{al}^{5}$ have also demonstrated that ELO can be used to fabricate high efficiency, low areal density, DJ-cells for space applications.

In this paper we will briefly describe our variant of the ELO process i.e. Metal-backed Epitaxial Lift Off (MELO), followed by a discussion of the growth and fabrication of a single junction GaAs PV cell. The results from this cell under 1-sun operation and under concentration will be described. Finally we will describe the growth, fabrication and test results of our first attempt at an inverted $\mathrm{InGaP} / \mathrm{GaAs}$ dual junction MELO cell. 


\section{EPITAXIAL LIFT OFF}

The principle of the ELO process that we are using is illustrate,for a single GaAs cell, in the process flow shown in Figure 1.

The epitaxial layers and AlAs release layers are first deposited epitaxially on a GaAs substrate, a thick $(>20 \mu \mathrm{m}) \mathrm{Cu}$ layer is electroplated on the surface of the wafer which acts a contact layer, support during processing and as a heat spreader during operation. The combination of the solar cell and electroplated $\mathrm{Cu}$ layer is referred to as the MELO platform.

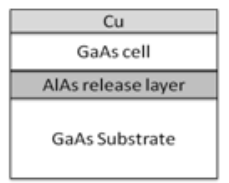

(a)
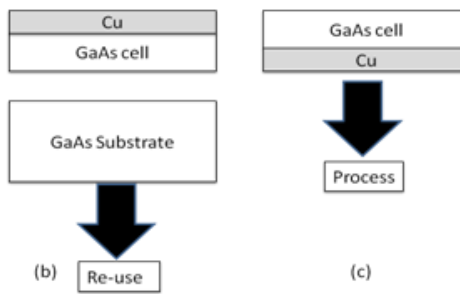

FIGURE 1: Schematic of the MELO process. (a) GaAs cell and release layer are deposited epitaxially and a thick $\mathrm{Cu}$ layer is electroplated on the wafer surface, (b) the release layer is removed, and (c) layer is inverted and processed into PV cells.

The AlAs release layer is removed by etching in HF using the method described by Schermer ${ }^{6}$. Following release of the solar cell, the GaAs substrate is available for further use and the solar cell and metal backing layer are inverted for further processing.

\section{SINGLE JUNCTION CELLS}

In a stepwise approach toward the realization of an inverted triple junction cell on the MELO platform we have first grown an inverted (SJ) photovoltaic cell to establish the basic layer release process and cell processing steps.

Single junction GaAs cells were grown by MOCVD at 50mbar on 2" GaAs (100) substrates misoriented by $6^{\circ}$ toward (111)A. The inverted cell structure is grown on a $10 \mathrm{~nm}$ AlAs release layer.

\section{MELO Cell Processing and 1-sun Measurements}

Following the electroplating with $\mathrm{Cu}$ the structure was mounted on a flexible carrier, attached to a cylinder and etched using HF. The released epitaxial layer was then inverted and processed into test solar cells $^{2}$. The top surface of the cell was not antireflection coated. 1-sun results from this MELO cell are shown in below.

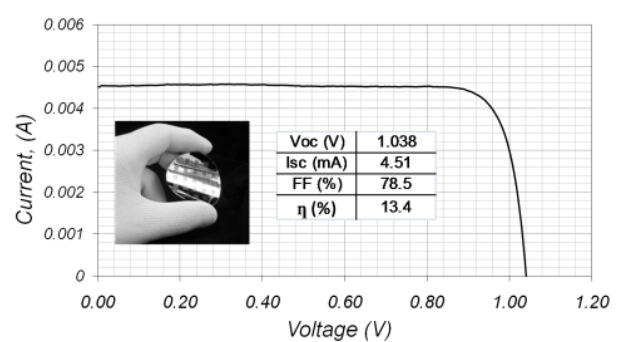

FIGURE 2: I-V curve for a MELO SJ GaAs photovoltaic cell under 1-sun illumination.

Figure 2 shows the measured I-V curve of an inverted SL MELO cell under illumination by an AM1.5G spectrum. The efficiency of this cell at $13.4 \%$ is sufficiently good for us to suggest and that the MELO process has not adversely compromised the PV cell's performance, especially when one considers that the cell is not AR coated (which will increase the efficiency by a relative $30 \%$ ) and that the GaAs base region of the structure was unusually thick at $1000 \mathrm{~nm}$. No attempt was made to optimize the performance of the cells through subsequent further growths.

\section{MELO Cells For CPV Applications}

A new process mask was made which allowed the testing of the MELO cells under concentration. Cells are $\sim 5 \mathrm{~mm} \times 4 \mathrm{~mm}$ in size and have a top contact and grid finger pattern suitable for use under concentration. To facilitate an evaluation of how well the SJ MELO GaAs cells would perform under concentration in a HCPV system the cells were singulated and die attached using a Ag-loaded epoxy to an example of the Al-pcb submount designed for use in Circadian's HCVP system. Top contacts were made to the PV cell by either ribbon bonding using Al-ribbon or by wire bonding with $\mathrm{Au}$-wires. Cells were tested at ambient temperature and flash-tested to $\sim 1400 x$. A cell attached to a pcb and wire bonded is shown in Figure 3.

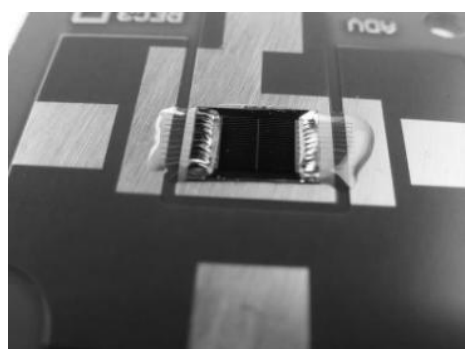

FIGURE 3: A single junction GaAs MELO PV cell that has been die attached to a pcb. Top contacts are made using gold wire bonds which are encapsulated for protection against mechanical damage. 
Results from a SJ GaAs cell under concentration are shown in Figures 4 and 5.

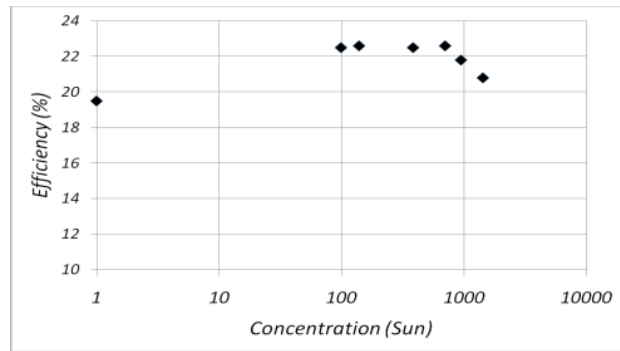

FIGURE 4: Variation of the efficiency of a MELO SJ GaAs cell versus concentration of sunlight.

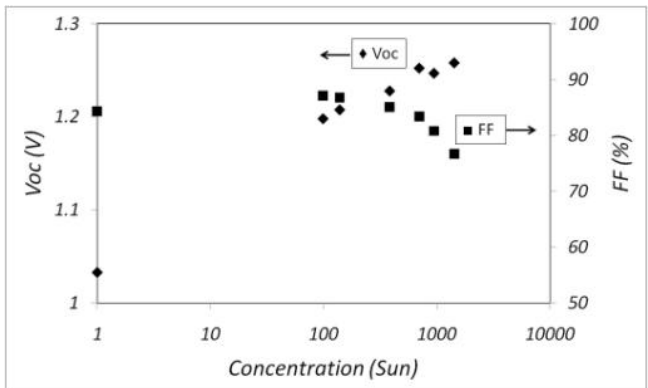

FIGURE 5: Variation of the Voc and FF of a MELO SJ GaAs cell versus concentration of sunlight.

Figure 4 shows the expected increase in efficiency of the SJ GaAs PV cell as the concentration increases until eventually $(>500 x)$ the increase in efficiency is offset by the decrease due to the series resistance of the cell.

This is further illustrated by the behaviour of Voc and FF versus concentration, as shown in Figure 5.

At a typical CPV system concentration of 500x the SJ cell has performance characteristics of a fill factor (FF) of $\sim 85 \%$, an efficiency $(\eta)$ of $\sim 22.5 \%$ and an open circuit voltage $\left(\mathrm{V}_{\mathrm{oc}}\right)=1.25 \mathrm{~V}$.

Whilst there is clearly room for improvement in device performance it should be noted that no attempt has been made to optimize either series resistance or the cell's efficiency and the structure has been used solely as a vehicle to demonstrate the feasibility of using MELO cells in HCPV systems.

\section{Inverted Dual Junction MELO Cells}

The next step in the realization of an inverted triple junction cell using the MELO platform is to grow and process an inverted dual junction $\mathrm{GaAs} / \mathrm{InGaP}$ PV cell. A schematic of the inverted dual junction (DJ) is shown in Figure 6.

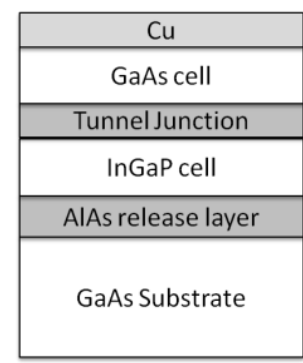

FIGURE 6: Schematic of an inverse dual junction GaAs/InGaP PV cell.

As a pre-requisite it is necessary to develop the growth of a Tunnel Junction (TJ) that would be capable of supporting currents equivalent to operation at $>1000 x$. This is non-trivial since the "inverse" growth involves growing an $\mathrm{AlGaAs} / \mathrm{GaAs} \mathrm{TJ}$ on an (AlGaIn)P back surface field (BSF) layer where the growth conditions are quite different for the Pcontaining and As-containing layers.

To optimize the TJ we have grown test structures of p++-AlGaAs (18nm) and n++-GaAs layers on an AlInGaP BSF layer $(30 \mathrm{~nm})$ capped with a n-AlGaInP $(30 \mathrm{~nm})$ window layer, both without and with a "thermal load". The "thermal load" is a thick layer of GaAs which is grown for a time equivalent to growing the GaAs cell following growth of the TJ. The n-type dopant is Si and the p-type dopant is C.

In Table 1, we have collected together the results of TJ test structures.

TABLE 1. Collected results from various Tunnel Junction test structures

\begin{tabular}{ccc}
\hline Structure & $\begin{array}{c}\text { Peak } \\
\text { Current } \\
\text { Density }\left(\mathbf{A} / \mathbf{c m}^{2}\right)\end{array}$ & Conc. \\
\hline $\begin{array}{c}\text { Non Inverse TJ } \\
\text { thermal load }\end{array}$ & 65 & $4260 \mathrm{x}$ \\
Inverse TJ & 33 & $2400 \mathrm{x}$ \\
Inverse TJ + & 21.5 & $1500 \mathrm{x}$ \\
thermal load & & \\
\hline
\end{tabular}

From Table 1 it is clear that we have arrived at a combination of layer structure and growth conditions that makes it possible to fabricate a TJ that will support currents equivalent to those at $\sim 1500 x$. It is also clear from this table that there is a significant degradation of the peak current density of the TJ. We postulate that the degradation when going from noninverse to inverse is due to the problems associated with growth of the As on $\mathrm{P}$ interface as opposed to the more conventional $\mathrm{P}$ on As. In the inverted structure the growth temperature needed for the autodoping of the p++ layer is extremely low at 570C and the decomposition of $\mathrm{PH} 3$ at these temperatures is very low making the transition from the AlGaInP BSF to the TJ a difficult one to achieve with a high quality 
interface. The continued degradation that's seen when adding the thermal load is presumably due to dopant diffusion.

Now that we have all the building blocks in place we have grown a DJ GaAs/InGaP. The GaAs cell has an AlGaAs BSF and an AlInP window layer whilst the InGaP cell has an AlGaInP BSF and an AlInP window layer. The tunnel junction between the two cells is as described above. The whole structure is grown on an AlAs layer to facilitate the lift-off process. Following ELO the layer was processed into cells in the same way as for the SJ GaAs cells.

Initial, 1-sun, results on the DJ cells are shown in Figures 7 and 8 below.

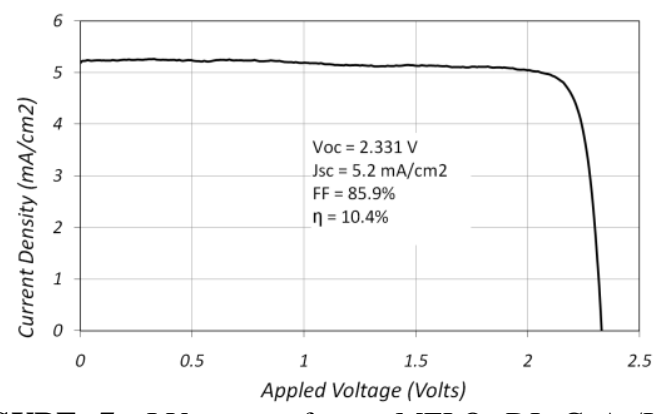

FIGURE 7: I-V curve for a MELO DJ GaAs/InGaP photovoltaic cell under 1-sun illumination

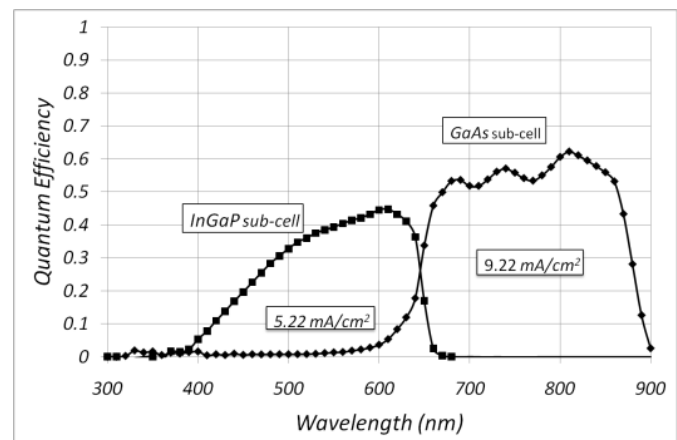

FIGURE 8: Quantum Efficiency measurement of a MELO DJ GaAs/InGaP photovoltaic cell.

These first 1-sun measurements are encouraging, showing good diode characteristics with a FF of $>85 \%$ and $\mathrm{a} \mathrm{V}_{\mathrm{oc}}$ of $2.33 \mathrm{~V}$. However, even taking into account the lack of an AR coating, which would boost the short circuit current by up to $30 \%$, and a grid pattern appropriate for measurements under concentration, the efficiency of the cell is low at $10.4 \%$.

Figure 8 shows the QE of the DJ cell. This plot clearly shows that the performance of the DJ cell is limited by the performance of the top InGaP cell. The root cause of this poor efficiency is unknown at the present time but is most likely due to a poor quality
AlInP window layer or a poor interface with the $\mathrm{InGaP}$ emitter which is acting as a source of recombination.

Further work is underway to try and improve the quality of the top InGaP cell.

\section{SUMMARY}

Progress in combining the ELO technique with a thick electroplated $\mathrm{Cu}$ support layer has been reported; the MELO process. The MELO process has been combined with inverted single junction cells successfully and we have also demonstrated SJ GaAs cells operating at concentrations up to $1400 \mathrm{x}$.

An inverted TJ has been developed that should be capable of providing an ohmic interconnect between sub-cells of a GaAs/InGaP DJ cell at $>100 x$. A first attempt at an inverted $\mathrm{GaAs} / \mathrm{InGaP}$ DJ cell has been successfully made with good diode characteristics but lower than desirable efficiency. The efficiency is currently limited by the performance of the top InGaP cell. Work to improve this is in progress.

\section{REFERENCES}

1. R.R. King, A. Boca, W. Hong, X-Q. Liu, D. Bhusari, D. Larrabee, K.M. Edmondson, D.C. Law, C.M. Fetzer, S. Mesropian and N.H. Karam, $24^{\text {th }}$ European Photovoltaic Solar Energy Conference, Hamburg, Germany, 2009, pp. 55-61

2. A. van Geelen, P.R. Hageman, G.J. Bauhuis, P.C. van Rijsingen, P. Schmidt and L.J. Gilling, Mater. Sci. and Eng. B45, 162 - 171 (1997)

3. G.J. Bauhuis, P. Mulder, E.J. Haverkamp, J.C.C. Huijben and J.J. Schermer, Solar Energy Materials \& Solar Cells 93, 1488-1491 (2009)

4. J.F. Geisz, D.J. Freidman, J.S. Ward, A. Duda, W.J. Olavarria, T.E. Moriarty, J.T. Kiehl, M.J. Romero, A.G. Norman and K.M. Jones, Appl. Phys. Letters 93, 123505 $1-123505-3$ (2008)

5. R. Tatavarti, G. Hillier, G. Martin, A Wibowo, R. Navaratnajarah, F. Tuminello, D. Hertkom, M. Disabb, C. Youtsey, D. McCallum and N.Pan, $34^{\text {th }}$ IEEE Photovoltaic Specialists Conference, Philadelphia, PA, 2009, pp. 002065 - 002067

6. J.J. Schermer, US Patent 6,9974,521 (2005)

${ }^{\text {i } E m a i l: ~ g e o f f r e y . d u g g a n ~ @ ~ c i r c a d i a n s o l a r . c o m ~}$ 